\title{
Effects of media and frame rates on hyperactivated bovine sperm motility and an analysis of sperm motility subpopula- tion structures in sex-sorted and non-sorted semen
}

\author{
Chihiro KANNO ${ }^{1}$, Sun-Sik KANG ${ }^{2}$, Kentaro Q. SAKAMOTO ${ }^{3}$, Yojiro YANAGAWA ${ }^{4}$, Seiji KATAGIRI ${ }^{4}$, and \\ Masashi NAGANO ${ }^{5 *}$
}

1 Laboratory of Clinical Veterinary Medicine for Large Animal, Division of Veterinary Medicine, School of Veterinary Medicine, Kitasato University, Towada 034-8628, Japan; c-kanno@vmas.kitasato-u.ac.jp

2 Hanwoo Research Institute, National Institute of Animal Science of Korea, 4937, Gyeonggang-ro, Daegwannyeong-myeon Pyeongchang-gun, Gangwon-do 25340, Korea; ricafo@vetmed.hokudai.ac.jp

3 Department of Marine Bioscience, Atmosphere and Ocean Research Institute, The University of Tokyo, 5-1-5 Kashiwanoha, Kashiwa 277-8564, Japan; kqsakamoto@g.ecc.u-tokyo.ac.jp

4 Laboratory of Theriogenology, Department of Veterinary Clinical Sciences, Graduate School of Veterinary Medicine, Hokkaido University, Kita-18, Nishi-9, Kita-ku, Sapporo 060-0818, Japan; yoji-y@vetmed.hokudai.ac.jp (Y. Yanagawa), katagiri@vetmed.hokudai.ac.jp (S. Katagiri)

5 Laboratory of Animal Reproduction, Department of Animal Science, School of Veterinary Medicine, Kitasato University, Towada 034-8628, Japan; mnaga@vmas.kitasato-u.ac.jp

* Correspondence: mnaga@vmas.kitasato-u.ac.jp;

Simple Summary: Capacitation is an important physiological change that occurs in sperm before they penetrate oocytes. However, sperm also exhibit capacitation-like changes during semen processing, which decreases fertility. Therefore, the evaluation of capacitation-like changes in frozenthawed sperm is essential for successful artificial insemination. We herein attempted to establish an objective method to evaluate the motility of bull sperm, particularly hyperactivation, a specific movement in capacitated sperm, using a computer-assisted sperm analysis. Sperm images captured at 150 frames per second (fps) showed a more detailed pathway than those at 30, 50, and $75 \mathrm{fps}$. The structures of sperm motility subpopulations in sex-sorted semen were examined as a low fertility semen model, and the results obtained showed that sex-sorted semen comprised more sperm with hyperactivation-like motility than non-sorted semen. Collectively, the present results indicate that a discriminant analysis has the ability to accurately describe differences in the structures of sperm motility subpopulations and may be useful for evaluations of semen fertility.

\begin{abstract}
We attempted to establish an objective method to accurately evaluate the motility of bull sperm and examined the effects of media for sperm suspensions and frame rates on data of computer-assisted sperm motility analysis (CASA). Sperm incubated in Brackett and Oliphant medium (BO) more clearly showed hyperactivation-like motility than those in synthetic oviductal fluid. Sperm images captured at 150 frames per second (fps) showed a trajectory that was closer to the real pathway than those at other frame rates $(30,50$, and $75 \mathrm{fps})$. We then examined the characteristics of sex-sorted and non-sorted semen using a cluster analysis followed by a discriminant analysis of sperm motility in BO at $150 \mathrm{fps}$. The results indicated that sex-sorted semen contained sperm with hyperactivation-like motility as the main subpopulation immediately after thawing and this subpopulation decreased after 2-h incubation. The main subpopulation in non-sorted semen had progressive motility that was maintained during incubation. In conclusion, usage of $\mathrm{BO}$ for sperm suspensions and capturing sperm motility at 150 fps by CASA were appropriate for evaluating bovine sperm motility. A discriminant analysis using data from a cluster analysis of motile sperm has the ability to accurately describe differences in the structures of sperm motility subpopulations.
\end{abstract}

Keywords: Capacitation; Computer-assisted sperm analysis; Hyperactivation; Sex-sorted semen; Sperm motility subpopulation 


\section{Introduction}

Capacitation is consisted of physiological changes in sperm before they penetrate oocytes [1]. Although the underlying mechanisms have not yet been elucidated in detail, several intracellular changes, such as increasing plasma membrane fluidity, cholesterol efflux, intracellular $\mathrm{Ca}^{2+}$ and cAMP concentrations, and protein phosphorylation, correlate with capacitation [2]. General treatments of semen, such as cryopreservation [3] and sexsorting [4], induce capacitation-like changes in sperm. Capacitation-like damage to bovine sperm during cryopreservation is one of the factors that decreases sperm fertility due to their short longevity $[5,6]$. Therefore, an evaluation of capacitation-like changes in frozenthawed sperm is important for successful artificial insemination (AI).

Fluorescent staining by chlortetracycline (CTC) [7] and Western blotting to assess protein phosphorylation [8] have been used to detect capacitation. However, these techniques may not be suitable as a routine evaluation in AI centers because observations of CTC staining require fluorescent microscopy and Western blotting is time-consuming. Therefore, the development of a convenient method for practitioners in AI centers to easily and accurately evaluate sperm capacitation is needed.

Capacitated sperm exhibit a specific movement that is recognized as hyperactivation. Sperm showing hyperactivation pass through the cumulus investment and zona pellucida of oocytes [9]. Although hyperactivation is captured by a computer-assisted sperm analysis (CASA) as increases in curvilinear velocity (VCL) and amplitude of lateral sperm head displacement (ALH) and decreases in linearity (LIN) and beat cross frequency (BCF) [9], there are currently no established criteria to evaluate the motility of hyperactivated sperm. A frame rate of 80-100 frames per second (fps) is recommended for the analysis of sperm capacitation in humans [10]. Although a frame rate of 30-60 fps is generally utilized in most CASA systems for cattle, a suitable frame rate for analyzing bovine hyperactivated sperm remains unclear.

Therefore, we herein examined the effects of the frame rate on the detection of hyperactivated sperm motility. We also investigated which of the following media were suitable for detecting hyperactivation: Brackett and Oliphant medium (BO) [11], commonly used for in vitro fertilization, and synthetic oviductal fluid (SOF) [12], frequently employed in in vitro cultures of bovine embryos. Furthermore, we assessed semen fertility using a cluster analysis followed by a discriminant analysis. Sex-sorted semen show lower fertility $[13,14]$ and shorter longevity than non-sorted semen [15]. Therefore, we examined the motility characteristics of sperm in sex-sorted semen as a model of low fertility sperm and compared them with those in non-sorted semen.

\section{Materials and Methods}

\subsection{Semen}

Non-sorted frozen semen derived from 6 Holstein bulls (A-F) and sex-sorted frozen semen derived from 3 Holstein bulls (D-F) donated by an AI center (Genetics Hokkaido, Kita-Hiroshima, Japan) were used in the present study. These semen were used commercially with proven acceptable conception rates by $\mathrm{AI}$ in the field.

\subsection{Sperm preparation}

Straws containing frozen semen were immersed in water at $37^{\circ} \mathrm{C}$ for $1 \mathrm{~min}$, and semen were expelled onto a 45/90\% Percoll layer diluted by BO $(112.00 \mathrm{mM} \mathrm{NaCl}, 4.02 \mathrm{mM}$ $\mathrm{KCl}, 0.83 \mathrm{mM} \mathrm{NaH}_{2} \mathrm{PO}_{4}, 2.25 \mathrm{mM} \mathrm{CaCl}_{2}, 0.52 \mathrm{mM} \mathrm{MgCl}_{2}, 37.00 \mathrm{mM} \mathrm{NaHCO}_{3}, 13.90 \mathrm{mM}$ glucose, $1.25 \mathrm{mM}$ sodium pyruvate, and $50 \mu \mathrm{g} / \mathrm{ml}$ gentamicin sulfate) or SOF $(107.70 \mathrm{mM}$ $\mathrm{NaCl}, 7.16 \mathrm{mM} \mathrm{KCl}, 1.19 \mathrm{mM} \mathrm{KH} \mathrm{PO}_{4}, 1.71 \mathrm{mM} \mathrm{CaCl}_{2}, 0.49 \mathrm{mM} \mathrm{MgCl}, 27.07 \mathrm{mM}$ Na$\mathrm{HCO}_{3}, 3.30 \mathrm{mM}$ sodium lactate, $0.33 \mathrm{mM}$ sodium pyruvate, $1.50 \mathrm{mM}$ glucose, and 50 $\mu \mathrm{g} / \mathrm{ml}$ gentamicin sulfate) without bovine serum albumin (BSA). Samples were then centrifuged at $700 \times \mathrm{g}$ for $20 \mathrm{~min}$ to select motile sperm. The supernatants were removed and 
the resulting sperm pellets were resuspended in media (BO or SOF) and centrifuged again at $500 \times \mathrm{g}$ for $5 \mathrm{~min}$ for washing. After a second centrifugation, the supernatants were removed, and sperm concentrations were calculated using a hemocytometer. Samples were diluted to $10 \times 10^{6}$ cells $/ \mathrm{ml}$ by BO or SOF containing $3.0 \mathrm{mg} / \mathrm{ml}$ BSA.

\subsection{Evaluation of sperm motility and sperm motility parameters by CASA}

Sperm motility was evaluated as described in a previous study [16]. Briefly, semen samples were introduced into a 20- $\mu$ m-deep chamber (SC20-01-04-B, Leja, GN NieuwVennep, Netherlands) preliminary warmed at $37^{\circ} \mathrm{C}$ on a hot plate (Kitazato Corporation, Shizuoka, Japan), and sperm motility was evaluated using a CASA system (SMAS, DITECT, Tokyo, Japan) based on the digitalized images obtained by a $\times 10$ negative-phase contrast microscope (E200, Nikon, Tokyo, Japan). The percentage of motile sperm was recorded and the following kinetic parameters were analyzed: straight line velocity (VSL: the straight-line distance from the beginning to end of a sperm track for $1 \mathrm{sec}), \mathrm{VCL}$, average path velocity (VAP: the average path velocity of sperm for $1 \mathrm{sec}$ ), ALH, and BCF. The CASA system recorded at $150 \mathrm{fps}$ and sperm with more than 120 frames were used in the analysis. The number of sperm analyzed per sample was at least 100 (sex-sorted) or 200 (non-sorted), including immotile sperm. LIN (=VSL/VCL) and straightness $(\mathrm{STR}=\mathrm{VSL} / \mathrm{VAP})$ were calculated automatically by the CASA system.

\subsection{Assessment of sperm motility subpopulations by a cluster analysis}

A cluster analysis of sperm motility was performed as described in a previous study [16]. Briefly, sperm kinetic parameters were obtained at $150 \mathrm{fps}$ by the CASA system, and VSL, VCL, VAP, ALH, and BCF were used as parameters in the cluster analysis. We assessed the number of clusters based on the shape of the dendrogram according to Ward's method. A multivariate k-means cluster analysis was then performed to classify the evaluated sperm into subpopulations based on motility variables.

\subsection{Evaluation of sperm motility subpopulation structures in sex-sorted and non-sorted semen by a discriminant analysis}

To evaluate the structures of sperm motility subpopulations, a discriminant analysis was performed using a custom written program (Igor Pro, Wavemetrics, Lake Oswego, OR, USA). Centroids and standard deviations in each cluster calculated by the cluster analysis were input into the program. Individual sperm were then categorized into each cluster by their motility parameters.

\subsection{Experimental design}

\subsubsection{Experiment 1: Effects of media and frame rates on hyperactivated sperm motility}

Non-sorted frozen semen derived from 3 bulls (A-C) were used. After the dilution of samples to $10 \times 106$ cells $/ \mathrm{ml}$ by BO or SOF, both samples were divided into two aliquots and stored in 1.5-ml microtubes for the induction of hyperactivation and for a control. Microtubes including samples were incubated in a dry bath at $37^{\circ} \mathrm{C}$. To induce hyperactivation [17], the calcium ionophore A23187 (final concentration $1 \mu \mathrm{M}$; C7522, Sigma-Aldrich, St. Louis, USA) was added to the samples. The calcium ionophore stock was 3.82 mM A23187 in ethanol. Sperm motility was evaluated using the CASA system before $(0$ $\min$ ) and after 1, 5, 10, 15, 20, 25, and 30 min with (+) or without (-) the A23187 treatment in each medium, $\mathrm{BO}(+)$ or (-) and $\mathrm{SOF}(+)$ or $(-)$, respectively. After the evaluation of sperm motility by CASA, the effects of media on motility parameters related to hyperactivation (VSL, VCL, LIN, ALH, and BCF) were investigated.

Since the $\mathrm{BO}(+)$ group clearly exhibited hyperactivation-like motility, the frame rate of data obtained was converted from 150 to 30, 50, and 75 fps using software (Frame step motility, DITECT). We then recalculated motility parameters related to hyperactivation (VSL, VCL, LIN, ALH, and BCF) at each frame rate.

\subsubsection{Experiment 2: Cluster analysis of sperm motility in sex-sorted and non-sorted sperm}


To make a cluster model, motility parameters derived from the $\mathrm{BO}(+)$ and (-) groups in Experiment 1 were used as reference data. The dendrogram described by Ward's method using the data of 44,570 motile sperm derived from BO samples (288 samples; 3 bulls, 6 replicates, with or without A23187, and each time point of incubation) is shown in Figure. 1. The number of clusters based on the shape of the dendrogram was 6 . The kinetic parameters of sperm in each cluster are shown in Table 1. Each cluster was numbered from the largest to smallest depending on the VSL value. Cluster 1 showed the highest VAP, the second highest VCL and LIN, and the third highest ALH and BCF. Cluster 2 showed the second highest STR, the third highest VCL, the highest BCF and LIN, but the second lowest ALH and lower VAP than clusters 1, 3, and 4. Cluster 3 had the highest VCL and ALH, the second highest VAP, and the third lowest BCF and LIN. Cluster 4 had the second highest VCL and the third highest ALH, BCF, and LIN, whereas VAP was lower than in clusters 1,2, and 3. Cluster 5 showed the second lowest values in all parameters, except for ALH. Cluster 6 had the lowest values in all parameters. The effects of A23187 on the structures of sperm motility subpopulations were evaluated.

In the experiment, motility parameters were obtained from sex-sorted and nonsorted sperm derived from bulls D-F, and 2,994 sperm data were (3 replicates in each group) fit to the model. A sperm treatment for sex-sorted and non-sorted semen was performed as described previously. Briefly, motile sperm separated using the $45 / 90 \%$ Percoll layer and recovered motile sperm were incubated in 50- $\mu$ l droplets of BO (final concentration of $10 \times 106$ cells $/ \mathrm{ml}$ ) under $5 \% \mathrm{CO} 2,5 \% \mathrm{O} 2$, and $90 \% \mathrm{~N} 2$ at $39^{\circ} \mathrm{C}$. After $0-$, $2-$, and 4-h incubations, sperm motility was analyzed by the CASA system at $37^{\circ} \mathrm{C}$.

\subsection{Statistical analysis}

All analyses were performed using JMP pro 14 (SAS, NC, USA). Data for each bull were pooled before the analysis. Data were analyzed by a repeated measures two-way ANOVA followed by Tukey-Kramer's HSD test or the Student's t-test. Data are shown as the mean \pm SD. Differences were considered to be significant at $\mathrm{P}<0.05$.

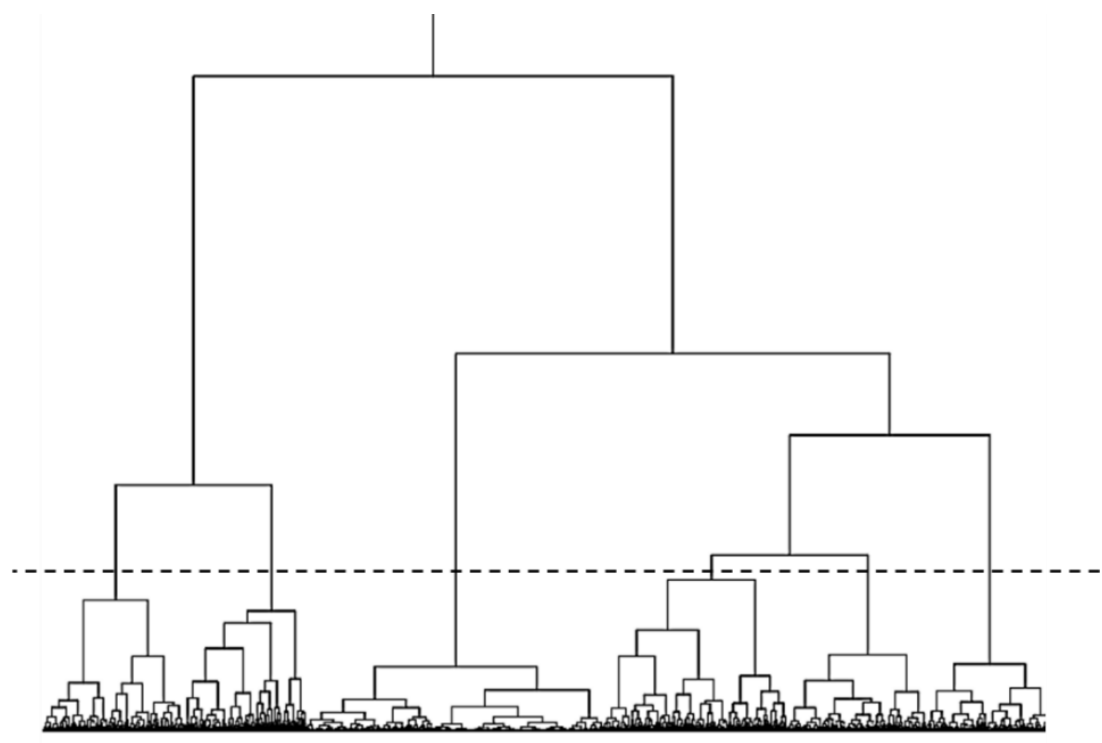

Figure 1. Dendrogram described by Ward's method to assess the number of clusters. 
To describe the dendrogram, 44,570 motile sperm incubated in BO were used.

Five independent motility variables: VSL $(\mu \mathrm{m} / \mathrm{sec})$, straight line velocity; VCL ( $\mu \mathrm{m} / \mathrm{sec})$, curvilinear velocity; LIN (VCL/VSL, \%), linearity; ALH ( $\mu \mathrm{m})$, amplitude of lateral sperm head displacement; BCF (Hz), beat cross frequency, were used as parameters.

A broken line was inserted to evaluate the number of clusters for the k-means method to categorize sperm.

Table 1. Kinetic parameters of sperm in each cluster

\begin{tabular}{|c|c|c|c|c|c|c|c|}
\hline Cluster & $\begin{array}{l}\text { No. of } \\
\text { sperm }\end{array}$ & $\begin{array}{c}\text { VSL } \\
(\mu \mathrm{m} / \mathrm{sec})\end{array}$ & $\begin{array}{c}\text { VCL } \\
(\mu \mathrm{m} / \mathrm{sec})\end{array}$ & $\begin{array}{c}\text { VAP } \\
(\mu \mathrm{m} / \mathrm{sec})\end{array}$ & $\begin{array}{l}\text { ALH } \\
(\mu \mathrm{m})\end{array}$ & $\begin{array}{l}\text { BCF } \\
(\mathrm{Hz})\end{array}$ & $\begin{array}{l}\text { LIN } \\
(\%)\end{array}$ \\
\hline 1 & 6032 & $149.2 \pm 27.9$ & $319.0 \pm 61.2$ & $163.2 \pm 21.4$ & $4.0 \pm 1.2$ & $21.8 \pm 6.2$ & $47.9 \pm 9.9$ \\
\hline 2 & 9397 & $80.7 \pm 28.2$ & $168.9 \pm 47.0$ & $94.1 \pm 27.0$ & $1.6 \pm 0.7$ & $28.3 \pm 5.8$ & $49.2 \pm 14.4$ \\
\hline 3 & 2671 & $77.9 \pm 45.9$ & $391.9 \pm 68.5$ & $145.1 \pm 29.9$ & $6.9 \pm 1.3$ & $8.4 \pm 4.7$ & $20.2 \pm 11.9$ \\
\hline 4 & 5203 & $52.0 \pm 29.5$ & $238.4 \pm 54.8$ & $90.9 \pm 27.1$ & $4.3 \pm 1.2$ & $10.6 \pm 5.8$ & $23.0 \pm 13.6$ \\
\hline 5 & 7792 & $20.9 \pm 15.9$ & $123.9 \pm 45.2$ & $35.6 \pm 17.6$ & $2.6 \pm 1.0$ & $7.5 \pm 4.5$ & $16.6 \pm 11.6$ \\
\hline 6 & 13475 & $5.4 \pm 5.9$ & $42.5 \pm 22.1$ & $9.9 \pm 7.8$ & $0.7 \pm 0.5$ & $6.9 \pm 3.6$ & $12.3 \pm 8.5$ \\
\hline
\end{tabular}

Values are the mean \pm SD.

VSL $(\mu \mathrm{m} / \mathrm{sec})$, straight line velocity; VCL ( $\mu \mathrm{m} / \mathrm{sec})$, curvilinear velocity; VAP $(\mu \mathrm{m} / \mathrm{sec})$, average path velocity; $\mathrm{ALH}(\mu \mathrm{m})$, amplitude of lateral sperm head displacement; BCF (Hz), beat cross frequency; LIN (VCL/VSL, $\%)$, linearity. 


\section{Results}

\subsection{Experiment 1}

Interactions between the effects of media and times after the A23187 treatment were observed for all kinetic parameters. As shown in Figure. 2, BO (+) showed the lowest values in VSL at $15-30 \mathrm{~min}(\mathrm{P}<0.05)$ and in LIN at all time points $(\mathrm{P}<0.05)$. BO $(+)$ showed higher values in VCL than in SOF $(+)$ at $0 \mathrm{~min}(\mathrm{P}<0.05)$ and this value decreased to a similar value with $\mathrm{SOF}(+)$ at all incubation times. At all incubation times, sperm in $\mathrm{BO}$ showed lower LIN than sperm in SOF $(\mathrm{P}<0.05)$, regardless of the A23187 treatment. SOF $(+)$ showed stable VCL during the incubation.

As shown in Figure. 3, ALH in $\mathrm{BO}(+)$ and SOF $(+)$ were not affected by the incubation time. $\mathrm{BO}(+)$ had higher ALH values than $\mathrm{SOF}(+)$ at 0,1 , and $5 \mathrm{~min}(\mathrm{P}<0.05)$. No significant difference was observed in ALH between $\mathrm{BO}(-)$ and SOF (+) during the incubation. BCF was lower in $\mathrm{BO}(+)$ and SOF $(+)$ than in $\mathrm{BO}(-)$ and SOF $(-)$ at $15-30 \mathrm{~min}(\mathrm{P}<0.05)$. No significant changes were observed in $\mathrm{BCF}$ in $\mathrm{BO}(-)$ or SOF (-) during the incubation. BCF was lower in $\mathrm{BO}(-)$ than in SOF $(-)$ at all incubation times $(\mathrm{P}<0.05)$.
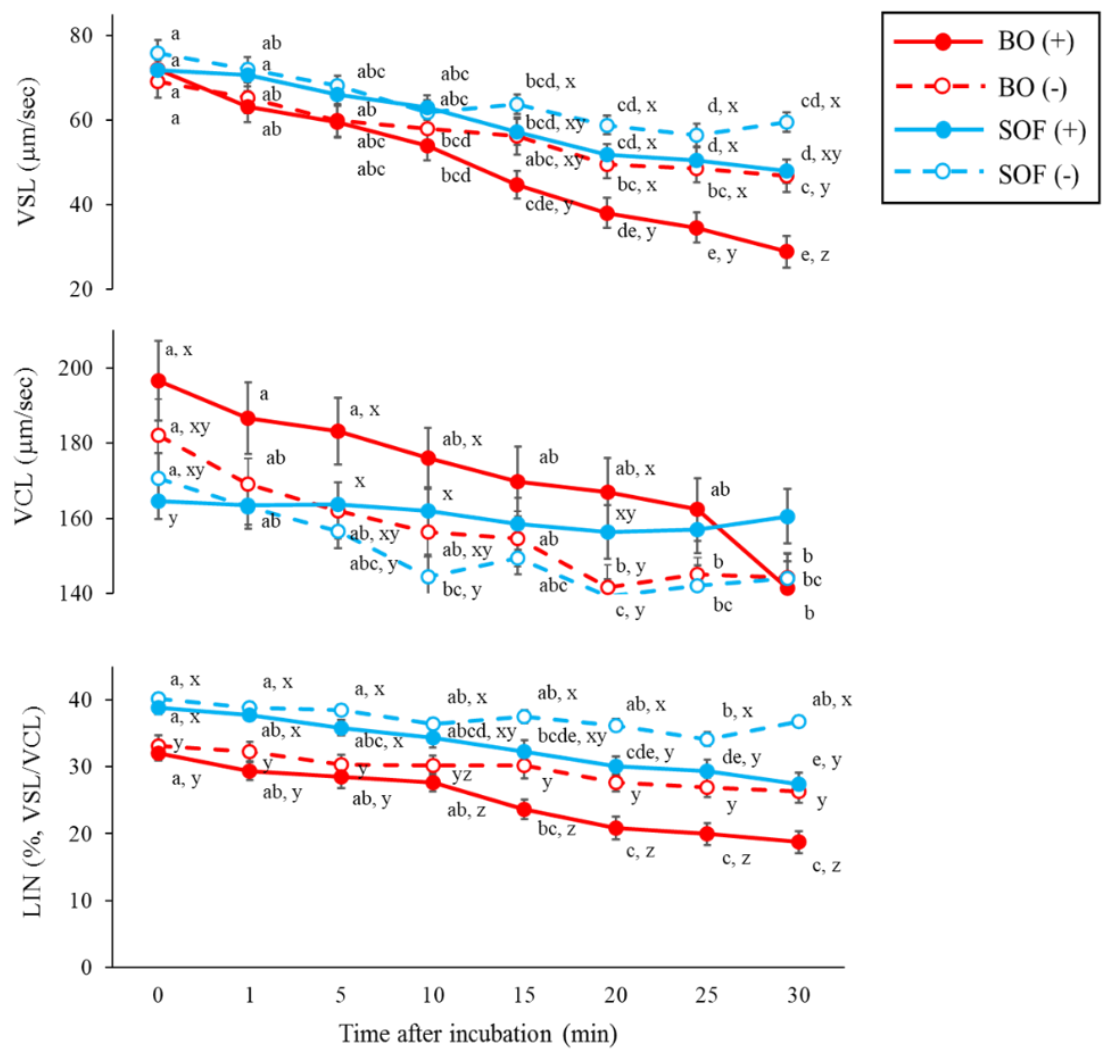

Figure 2. Effects of media on sperm motility

Three bulls were used and 6 replicates were performed in each group.

VSL ( $\mu \mathrm{m} / \mathrm{sec})$, straight line velocity; VCL ( $\mu \mathrm{m} / \mathrm{sec})$, curvilinear velocity; LIN (VCL/VSL, $\%)$, linearity.

a, b, c, d, e: Different letters indicate significant differences between time after the treatment $(\mathrm{P}<0.05)$.

$\mathrm{x}, \mathrm{y}, \mathrm{z}$ : Different letters indicate significant differences between groups $(\mathrm{P}<0.05)$. 

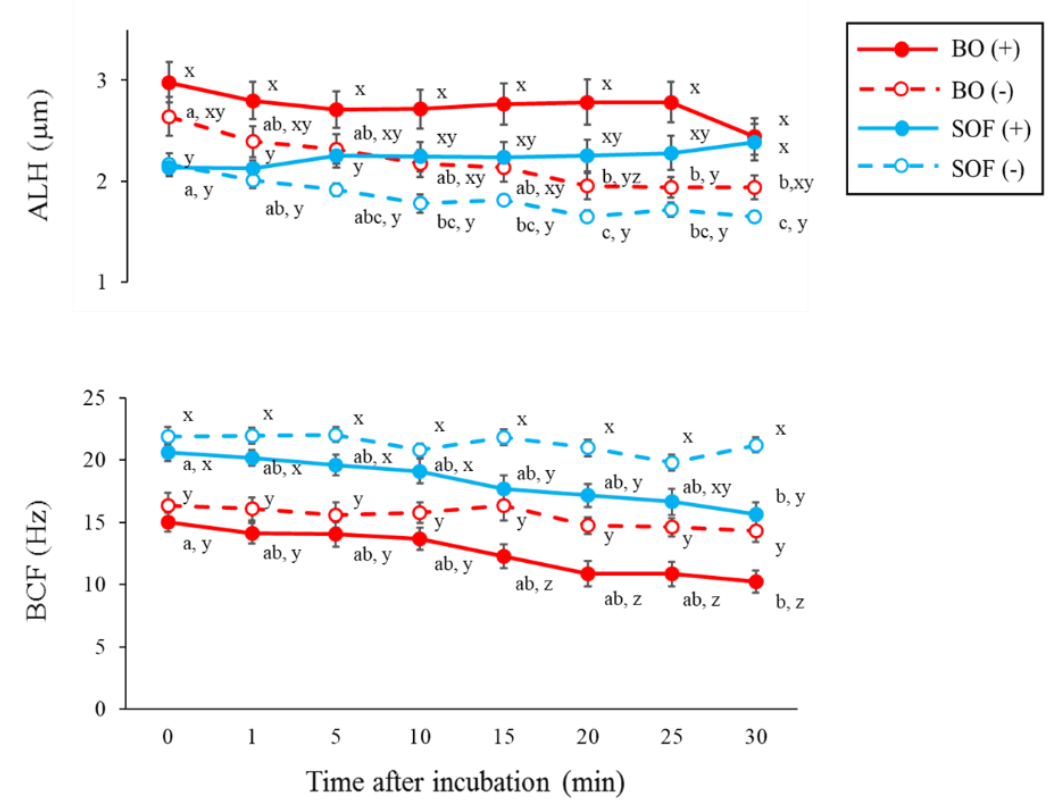

Figure 3. Effects of media on sperm head motility

Three bulls were used and 6 replicates were performed in each group.

$\mathrm{ALH}(\mu \mathrm{m})$, amplitude of lateral sperm head displacement; BCF $(\mathrm{Hz})$, beat cross frequency.

a, b, c; Different letters indicate significant differences between time after the treatment $(\mathrm{P}<0.05)$.

$x, y, z$; Different letters indicate significant differences between groups $(P<0.05)$.

Error bars indicate SEM.
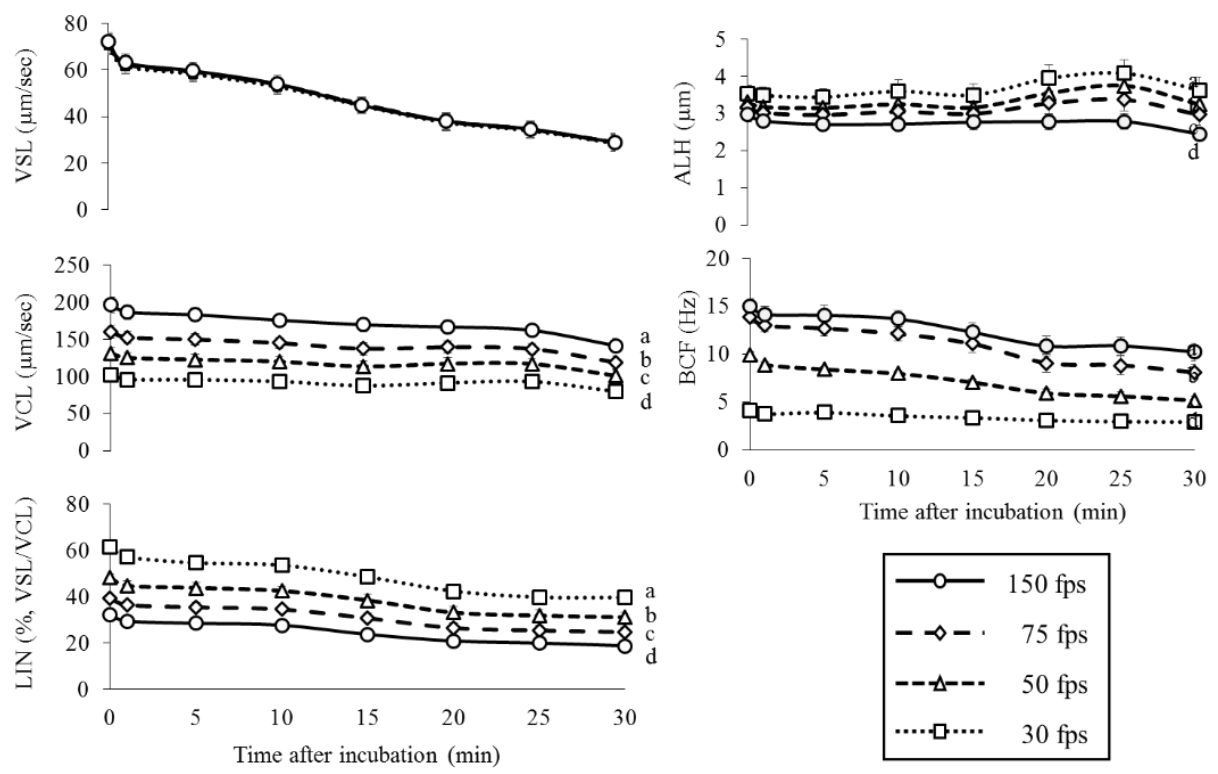

Figure 4. Effects of frame rates on sperm and head motility

Three bulls were used and 6 replicates were performed in each group.

VSL $(\mu \mathrm{m} / \mathrm{sec})$, straight line velocity; VCL $(\mu \mathrm{m} / \mathrm{sec})$, curvilinear velocity; LIN (VCL/VSL, $\%)$, linearity; $\mathrm{ALH}(\mu \mathrm{m})$, amplitude of lateral sperm head displacement; $\mathrm{BCF}(\mathrm{Hz})$, beat cross frequency.

$a, b, c, d$ : Different letters indicate significant differences between frame rates $(\mathrm{P}<0.05)$.

Error bars indicate SEM.

No interactions were noted between frame rates and incubation periods; therefore, data from different time points were pooled in each group for a comparison between groups with different frame rates. As shown in Figure. 4, VSL was similar at all frame 
rates. VCL and BCF significantly increased, whereas ALH and LIN significantly decreased as the frame rate became higher $(\mathrm{P}<0.05)$.

\subsection{Experiment 2}

The effects of A23187 on the structures of sperm motility subpopulations are shown in Figure. 5. The proportions of clusters 1 and 2 were lower, while those of clusters 3 and 4 were higher in sperm treated with A23187 than in those without the A23187 treatment $(\mathrm{P}<0.05)$.

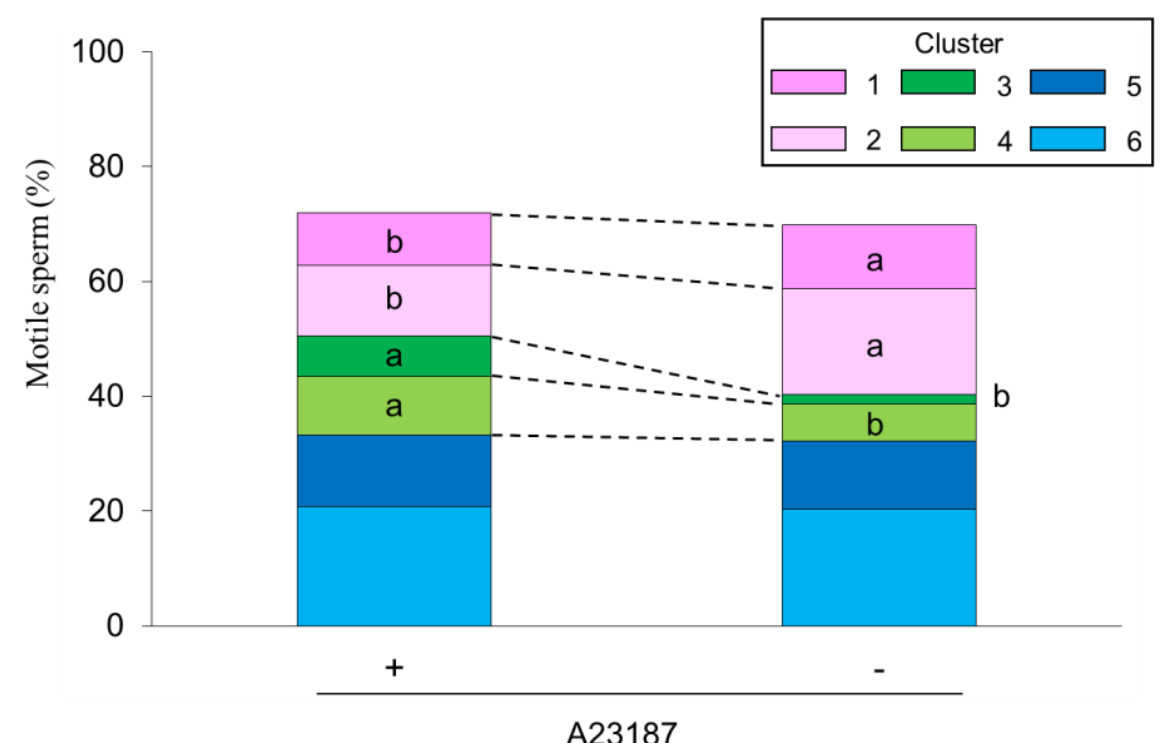

Figure 5. Effects of the calcium ionophore A23187 treatment on structures of sperm motility subpopulations

Data on sperm with (+) and without (-) the treatment at all time points were pooled. Three bulls were used and 6 replicates were performed in each group.

All time points (0-30 min) were pooled.

$\mathrm{a}, \mathrm{b}$; Different letters indicate significant differences in the same cluster between groups $(\mathrm{P}<0.05)$.

The sperm kinetic parameters of each cluster are shown in Table 1

The results of the discriminant analysis to evaluate the motility of sperm in sex-sorted semen are shown in Figure. 6. The proportion of cluster 1 was lower in sex-sorted than in non-sorted semen at all incubation times $(\mathrm{P}<0.05)$. The proportion of cluster 3 was higher in sex-sorted than in non-sorted semen immediately after thawing $(\mathrm{P}<0.05)$. Although the percentage of motile sperm decreased with the progression of the incubation $(\mathrm{P}<0.05)$, no reductions were observed in the proportion of cluster 1 between 0 and $2 \mathrm{~h}$ regardless of sorting. The percentages of motile sperm were lower in sex-sorted than in non-sorted semen at 2 and $4 \mathrm{~h}(\mathrm{P}<0.05)$. 


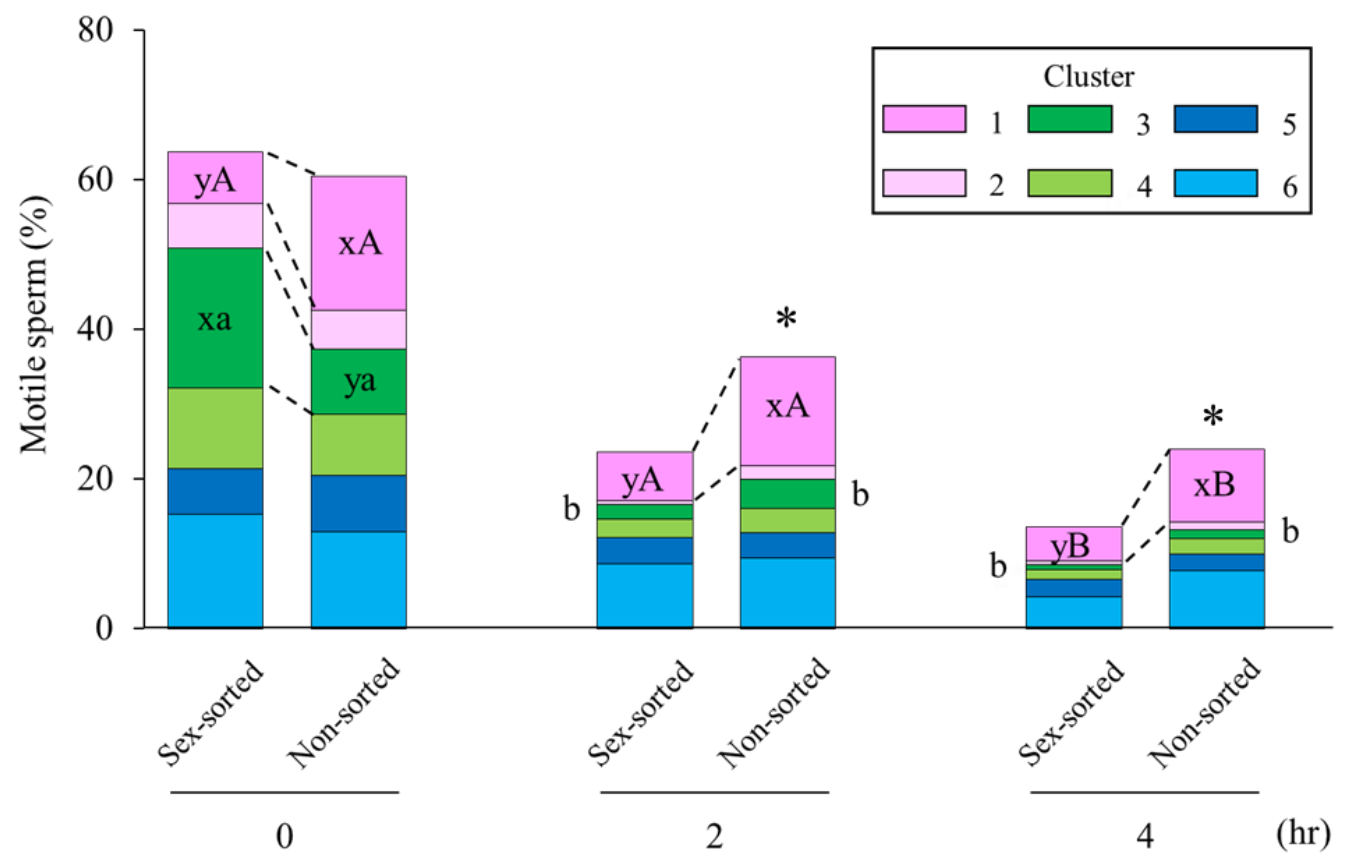

Figure 6. Effects of sex sorting and an incubation in $\mathrm{BO}$ on structures of sperm motility subpopulations

Three bulls were used and 3 replicates were performed in each group.

*; The asterisk indicates a significant difference in the percentage of motile sperm between sex- and non-sorted sperm $(\mathrm{P}<0.05)$.

$\mathrm{x}, \mathrm{y}$; Different letters indicate significant differences in the same cluster between sexand non-sorted sperm $(\mathrm{P}<0.05)$.

A, B; Different letters indicate significant differences in the proportion of cluster 1 between incubation times $(\mathrm{P}<0.05)$.

$a, b$; Different letters indicate significant differences in the proportion of cluster 3 between incubation times $(\mathrm{P}<0.05)$.

The sperm kinetic parameters of each cluster are shown in Table 1. 


\section{Discussion}

In the present study, sperm incubated in $\mathrm{BO}$ more clearly exhibited hyperactivationlike motility, i.e., high VCL, high ALH, low VLS, and low LIN, than those in SOF not only in the A23187 treatment group, but also in the control. Although calcium ionophores induce capacitation by increasing the intracellular influx of $\mathrm{Ca}^{2+}[2], \mathrm{Ca}^{2+}$ concentrations were similar in $\mathrm{BO}$ and SOF. On the other hand, $\mathrm{BO}$ contained a higher concentration of $\mathrm{HCO}_{3}^{-}$, an effector of capacitation [18]. The higher $\mathrm{HCO}_{3}^{-}$concentration in $\mathrm{BO}$ than in SOF may facilitate the induction of sperm hyperactivation. In the present study, frame rates affected sperm kinetic parameters evaluated by CASA, except for VSL. Changes were observed in sperm kinetic parameters at higher frame rates, namely, increases in VCL and ALH and decreases in LIN and BCF. These changes were consistent with previously reported hyperactivation-like changes [19]. These results suggest that sperm motility captured at higher frame rates shows a trajectory that is closer to the real pathway than that at a lower frame rate (Figure 7). Therefore, a lower frame rate cannot capture all sperm movement by dropping frames. Although 50-60 fps is recommended for the evaluation of human sperm motility by CASA [20], Mortimer et al. [21] suggested that ram sperm in culture medium need to be examined at no lower than $75 \mathrm{fps}$, with $100 \mathrm{fps}$ being preferable. Previous studies reported that boar [22] and stallion [23] sperm required higher frame rates (200-250 fps) than the conventional frame rate, such as 25 and $50 \mathrm{fps}$, to more accurately evaluate their motility. The trajectories of sperm with hyperactivation-like motility captured at $50 \mathrm{fps}$ was not smooth in the present study, and they were sometimes not evaluated as motile sperm (Figure 8). These results indicate that the images captured at 150 fps provide an accurate evaluation of the trajectory of sperm in cattle.
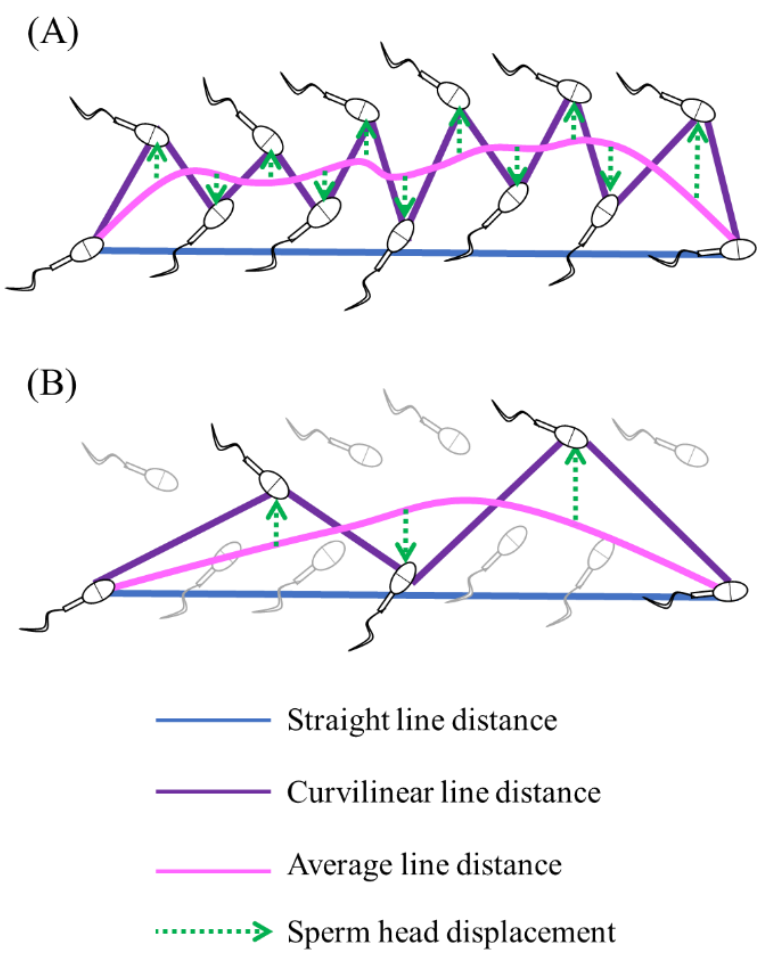

Figure 7. Differences in images of sperm trajectories between different frame rates (A) is a sperm trajectory captured at a 3-fold higher frame rate than (B). No significant difference was observed in the straight-line distance between (A) and (B). However, the curvilinear line distance, sperm head displacement, and the number of sperm crossing the average distance were increased at (A). Therefore, sperm kinetic parameters other than VSL were affected by the frame rate. 
(A)

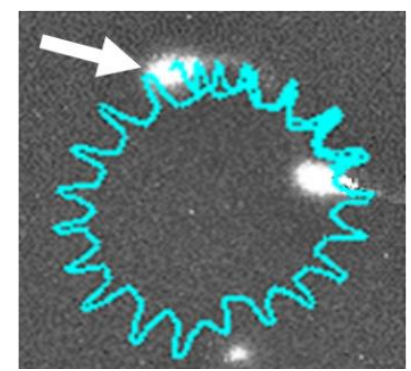

(B)

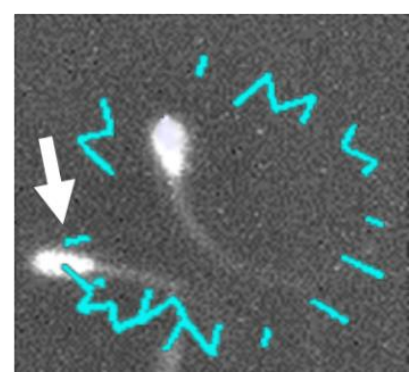

Figure 8. Trajectory of a sperm with hyperactivation-like motility at difference frame rates

Trajectories were described by capturing sperm (white arrows).

(A) A sperm with hyperactivation-like motility captured at $150 \mathrm{fps}$. The trajectory was smooth and without interruptions. (B) The same sperm with hyperactivationlike motility captured at $50 \mathrm{fps}$.

The trajectory was partly interrupted and not used to calculate sperm kinetic parameters by the CASA system.

The number of bovine sperm incubated in $\mathrm{BO}$ for clustering in the present study $(44,570$ motile sperm) was markedly higher than in previous studies $(16,730-23,585$ motile sperm) [24-27], and sperm in BO clearly exhibited hyperactivation-like characteristics; therefore, data on sperm captured at $150 \mathrm{fps}$ were used as reference data for the discriminant analysis. The cluster analysis categorized motile sperm into 6 clusters in the present study. Sperm in clusters 1 and 2 exhibited higher BCF and LIN than sperm in other clusters. In a previous study, sperm with high LIN maintained strong activity without hyperactivation $6 \mathrm{~h}$ after an incubation [28], and are regarded as progressively motile sperm, which is related to fertilization [25,29]. Clusters 3 and 4 included sperm with higher VCL and ALH and lower LIN and BCF than clusters 1 and 2. These values indicate a widely beating head in a circular motion, which is similar to the motility of hyperactivated sperm [30]. Moreover, sperm in clusters 3 and 4 were observed at a higher proportion in $\mathrm{BO}(+)$ than $\mathrm{BO}(-)$. Therefore, clusters 3 and 4 are subgroups of hyperactivated sperm. Although sperm in cluster 5 also showed low LIN, other parameters related to hyperactivation, VCL and ALH, were lower than those in clusters 3 and 4. In addition, sperm in cluster 6 showed very low values in all parameters. It means that sperm in clusters 5 and 6 are losing motility and cannot contribute to fertilization. The number of clusters in the present study (6 clusters) was higher than in previous studies that analyzed bull sperm motility subpopulations (3-4 clusters) [23-27,31]. We speculate that this difference may be due to variations in frame rates in the CASA system. In the present study, a detailed sperm pathway was described by capturing sperm motility at a high frame rate. Therefore, images captured at $150 \mathrm{fps}$ provide more information on the characteristics of sperm motility, and, as a consequence, sperm may be categorized into more clusters than those captured by a low frame rate.

In the present study, the discriminant analysis showed that the main subpopulations of sex-sorted and non-sorted semen were clusters 3 and 1, respectively, immediately after thawing. The proportion of cluster 1 was maintained until $2 \mathrm{~h}$ after thawing in both semen and was always lower in sex-sorted than in non-sorted semen after thawing. However, the proportion of cluster 3 decreased in both semen immediately after thawing. These results indicate that sperm exhibiting hyperactivation-like motility have shorter longevity than progressively motile sperm. In the present study, we used sperm derived from frozen-thawed sex-sorted semen as a model of low fertility sperm. Sex-sorted semen show lower fertility than non-sorted semen after AI [13,14] and also have shorter longevity [15]. Furthermore, the shorter lifespan of sperm in sex-sorted semen was previously proposed to be associated with capacitation-like changes induced by sex sorting [32]. The results of 
the present study clearly demonstrated that a higher proportion of sperm with hyperactivation-like motility was present in sex-sorted semen. This is the first study to apply a discriminant analysis to the evaluation of sperm motility and the results obtained showed that a cluster analysis is an effective method for assessing semen fertility after AI. However, the data size and types of sperm motility needed to establish valid reference data for the evaluation of sperm motility subpopulations remain unclear. Therefore, further studies are required to establish the best reference data size for evaluations of the structures of sperm motility subpopulations to predict semen fertility.

\title{
5. Conclusions
}

The use of BO for sperm suspensions and capturing sperm motility at $150 \mathrm{fps}$ by CASA were appropriate for evaluating sperm kinetics, including hyperactivation-like motility. In addition, a discriminant analysis using the data of a cluster analysis of motile sperm appropriately describes differences in the structures of sperm motility subpopulations in semen, and assesses the fertility of semen by describing the proportions of progressively motile and hyperactivation-like motile sperm.

\begin{abstract}
Author Contributions: Conceptualization, C. Kanno, S. Kang, Y. Yanagawa, and M. Nagano; Formal analysis, C. Kanno and K. Q. Sakamoto; Funding acquisition, C. Kanno and M. Nagano; Investigation, C. Kanno and S. Kang; Methodology, C. Kanno, S. Kang., K. Q. Sakamoto, Y. Yanagawa, and M. Nagano; Project administration, M. Nagano; Software, K. Q. Sakamoto, Supervision, S. Katagiri and M. Nagano; Validation, M. Nagano; Writing - original draft, C. Kanno; Writing - reviewing \& editing, C. Kanno, S. Kang, K. Q. Sakamoto, Y. Yanagawa, S. Katagiri, and M. Nagano. All authors have read and agreed to the published version of the manuscript.
\end{abstract}

Funding: This study was supported by JSPS KAKENHI Grant Number JP19H03117 to M. Nagano, JP21K14987 to C. Kanno, and the Kuribayashi scholarship foundation to C. Kanno.

Data Availability Statement: The data presented in the present study are available upon request from the corresponding author.

Acknowledgments: We are grateful to Genetics Hokkaido for the provision of frozen bull semen samples.

Conflicts of Interest: The authors declare no conflicts of interest.

\section{References}

1. Austin, C.R. The capacitation of the mammalian sperm. Nature 1952, 170, 326.

2. Breitbart, H. Intracellular calcium regulation in sperm capacitation and acrosomal reaction. Mol. Cell Endocrinol. 2002, 187, 139144.

3. Watson, P.F. Recent developments and concepts in the cryopreservation of spermatozoa and the assessment of their post-thawing function. Reprod. Fertil. Dev. 1995, 7, 871-891.

4. Mocé, E.; Graham, J.K.; Schenk, J.L. Effect of sex-sorting on the ability of fresh and cryopreserved bull sperm to undergo an acrosome reaction. Theriogenology 2006, 66, 929-936.

5. Harrison, R.A. Capacitation mechanisms, and the role of capacitation as seen in eutherian mammals. Reprod. Fertil. Dev. 1996, $8,581-594$.

6. Moce, E.; Graham, J.K. Cholesterol-loaded cyclodextrins added to fresh bull ejaculates improve sperm cryosurvival. J. Anim. Sci. 2006, 84, 826-833.

7. Saling, P.M.; Storey, B.T. Mouse gamete interactions during fertilization in vitro. Chlortetracycline as a fluorescent probe for the mouse sperm acrosome reaction. J. Cell Biol. 1979, 83, 544-555.

8. Galantino-Homer, H.L.; Visconti, P.E.; Kopf, G.S. Regulation of protein tyrosine phosphorylation during bovine sperm capacitation by a cyclic adenosine 3', 5'-monophosphate-dependent pathway. Biol. Reprod. 1997, 56, 707-719.

9. Suarez, S.S. Control of hyperactivation in sperm. Hum. Reprod. Update 2008, 14, 647-657.

10. Lu, J.C.; Huang, Y.F.; Lü, N.Q. Computer-aided sperm analysis: past, present and future. Andrologia 2014, 46, 329-338.

11. Brackett, B.G.; Oliphant, G. Capacitation of rabbit spermatozoa in vitro. Biol. Reprod. 1975, 12, $260-274$.

12. Tervit, H.R.; Whittingham, D.G.; Rowson, L.E.A. Successful culture in vitro of sheep and cattle ova. J. Reprod. Fertil. 1972, 30, 493-497. 
13. DeJarnette, J.M.; Leach, M.A.; Nebel, R.L.; Marshall, C.E.; McCleary, C.R.; Moreno, J.F. Effects of sex-sorting and sperm dosage on conception rates of Holstein heifers: is comparable fertility of sex-sorted and conventional semen plausible? J. Dairy Sci. 2011, 94, 3477-3483.

14. Hall, J.B.; Kasimanickam, R.K.; Glaze, J.B.; Roberts-Lew, M.C. Impact of delayed insemination on pregnancy rates to gender selected semen in a fixed-time AI system. Theriogenology 2017, 102, 154-161.

15. Beilby, K.H.; Grupen, C.G.; Thomson, P.C.; Maxwell, W.M.C.; Evans, G. The effect of insemination time and sperm dose on pregnancy rate using sex-sorted ram sperm. Theriogenology 2009, 71, 829-835.

16. Kanno, C.; Sakamoto, K.Q.; Yanagawa, Y.; Takahashi, Y.; Katagiri, S.; Nagano, M. Comparison of sperm subpopulation structures in first and second ejaculated semen from Japanese black bulls by a cluster analysis of sperm motility evaluated by a CASA system. J. Vet. Med. Sci. 2017, 79, 1359-1365.

17. Suarez, S.S.; Vincenti, L.; Ceglia, M.W. Hyperactivated motility induced in mouse sperm by calcium ionophore A23187 is reversible. J. Exp. Zool. 1987, 244, 331-336.

18. Harrison, R.A.P.; Ashworth, P.J.C.; Miller, N.G.A. Bicarbonate/CO2, an effector of capacitation, induces a rapid and reversible change in the lipid architecture of boar sperm plasma membranes. Mol. Reprod. Dev. 1996, 45, 378-391.

19. Castellini, C.; Dal Bosco, A.; Ruggeri, S.; Collodel, G. What is the best frame rate for evaluation of sperm motility in different species by computer-assisted sperm analysis? Fertil. Steril. 2011, 96, 24-27.

20. Morris, A.R.; Coutts, J.R.T.; Robertson, L. A detailed study of the effect of videoframe rates of 25, 30 and 60 Hertz on human sperm movement characteristics. Hum. Reprod. 1996, 11, 304-310.

21. Mortimer, S.T.; van der Horst, G.; Mortimer, D. The future of computer-aided sperm analysis. Asian J. Androl. 2015, 17, 545553.

22. Valverde, A.; Madrigal, M.; Caldeira, C.; Bompart, D.; de Murga, J.N.; Arnau, S.; Soler, C. Effect of frame rate capture frequency on sperm kinematic parameters and subpopulation structure definition in boars, analysed with a CASA-Mot system. Reprod. Dom. Anim. 2019, 54, 167-175.

23. Gacem, S.; Bompart, D.; Valverde, A.; Catalán, J.; Miró, J.; Soler, C. Optimal frame rate when there were stallion sperm motility evaluations and determinations for kinematic variables using CASA-Mot analysis in different counting chambers. Anim. Reprod. Sci. 2020, 223, 106643.

24. Ferraz, M.; Morató, R.; Yeste, M.; Arcarons, N.; Pena, A.I.; Tamargo, C.; Hidalgo, C.O.; Muiño, R.; Mogas, T. Evaluation of sperm subpopulation structure in relation to in vitro sperm-oocyte interaction of frozen-thawed semen from Holstein bulls. Theriogenology 2014, 81, 1067-1072.

25. Muiño, R.; Tamargo, C.; Hidalgo, C.O.; Peña, A.I. Identification of sperm subpopulations with defined motility characteristics in ejaculates from Holstein bulls: effects of cryopreservation and between-bull variation. Anim. Reprod. Sci. 2008, 109, 27-39.

26. Muiño, R.; Peña, A.I.; Rodríguez, A.; Tamargo, C.; Hidalgo, C.O. Effects of cryopreservation on the motile sperm subpopulations in semen from Asturiana de los Valles bulls. Theriogenology 2009, 72, 860-868.

27. Muiño, R.; Peña, A.I.; Quintela, L.A.; Becerra, J.; Herradón, P.; Gonzalez, F.H. Management of the storage of cryopreserved sperm on dairy cattle farms. Biotechnol. Anim. Husb. 2015, 31, 85-100.

28. Kang, S.; Koyama, K.; Huang, W.; Yang, Y.; Yanagawa, Y.; Takahashi, Y.; Nagano, M. Addition of D-penicillamine, hypotaurine, and epinephrine (PHE) mixture to IVF medium maintains motility and longevity of bovine sperm and enhances stable production of blastocysts in vitro. J. Reprod. Dev. 2015, 61, 99-105.

29. Larsen, L.; Scheike, T.; Jensen, T.K.; Bonde, J.P.; Ernst, E.; Hjollund, N.H.; Zhou, Y.; Skakkebæk, N.E.; Giwercman, A.; Danish First Pregnancy Planner Study, T. Computer-assisted semen analysis parameters as predictors for fertility of men from the general population. Hum. Reprod. 2000, 15, 1562-1567.

30. Ho, H.-C.; Suarez, S.S. Hyperactivation of mammalian spermatozoa: function and regulation. Reproduction 2001, 122, 519-526.

31. Yániz, J.L.; Palacín, I.; Caycho, K.S.; Soler, C.; Silvestre, M.A.; Santolaria, P. Determining the relationship between bull sperm kinematic subpopulations and fluorescence groups using an integrated sperm quality analysis technique. Reprod. Fertil. Dev. 2018, 30, 919-923.

32. Maxwell, W.M.C.; Johnson, L.A. Chlortetracycline analysis of boar spermatozoa after incubation, flow cytometric sorting, cooling, or cryopreservation. Mol. Reprod. Dev. 1997, 46, 408-418. 